\title{
Substitution of anti-androgens and tegafur-uracil combination therapy for castration-resistant prostate cancer: Results of a multi-center randomized phase II study
}

\author{
MASAYUKI TAKAHASHI ${ }^{1}$, RUMI KAWABATA ${ }^{2}$, AKIRA KAWANO $^{3}$, YOSHIHIDE MURAKAMI $^{4}$, \\ YASUSHI SUTOU ${ }^{5}$, TOHRU INAI ${ }^{6}$, SEIJI AKAZAWA ${ }^{7}$, TAKUMI HAMAO ${ }^{8}$, HIDEKI HAYASHI ${ }^{9}$, \\ TOMOYA FUKAWA ${ }^{1}$, MASAHIKO TAKEMURA ${ }^{1}$, YASUYO YAMAMOTO ${ }^{1}$, KUNIHISA YAMAGUCHI ${ }^{1}$, \\ HIROFUMI IZAKI ${ }^{1}$, TOMOHARU FUKUMORI ${ }^{1}$ and HIROOMI KANAYAMA ${ }^{1}$

\begin{abstract}
${ }^{1}$ Department of Urology, Institute of Health Biosciences, The University of Tokushima Graduate School, Tokushima 770-8503; ${ }^{2}$ Tokushima Research Center, Taiho Pharmaceutical Co. Ltd., Tokushima 771-0194; ${ }^{3}$ Department of Urology, Sanuki Municipal Hospital, Sanuki 769-2393; ${ }^{4}$ Department of Urology, Tokushima Municipal Hospital, Tokushima 770-0812; ${ }^{5}$ Department of Urology, Tsurugi Municipal Handa Hospital, Mima 779-4401; ${ }^{6}$ Department of Urology, Tokushima Prefectural Central Hospital, Tokushima 770-8539; ${ }^{7}$ Department of Urology, Tokushima Prefecture Naruto Hospital, Naruto 772-8503;

${ }^{8}$ Department of Urology, Kamei Hospital, Tokushima 770-8070; ${ }^{9}$ Department of Urology, Oe Kyodou Hospital, Yoshinogawa 776-8511, Japan
\end{abstract}

Received February 23, 2013; Accepted April 12, 2013

DOI: 10.3892/ijo.2013.1997

\begin{abstract}
We conducted this study to determine whether substitution with anti-androgen (SOA) and tegafur-uracil (a pro-drug of 5-FU) combination therapy is more effective than SOA alone after relapse from initial hormonal therapy. Patients who were histologically confirmed and relapsed after initial hormonal therapy were included. All patients were randomly allocated into two groups: SOA alone (group A) or SOA combined with tegafur-uracil (group B). The mRNA expression of four enzymes, including thymidylate synthase (TS), dihydropyrimidine dehydrogenase (DPD), orotate phosphoribosyltransferase (OPRT) and thymidine phosphorylase (TP), in prostate cancer cells was analyzed by quantitative reverse-transcription polymerase chain reaction. Fifty-two patients were enrolled in this study. The median age was 77 (range: 47-92) years. The PSA response rate in group B $(61.5 \%)$ tended to be higher compared to that in group A (34.6\%) $(\mathrm{p}=0.095)$. Group B (median: 15.9 months) had a significantly longer time to PSA progression (TTP) compared to group A $(6.4$ months $)(\mathrm{p}=0.014)$. In patients with a lower TS expression or a higher OPRT expression, group B demonstrated a higher PSA response rate compared to group A
\end{abstract}

Correspondence to: Dr Masayuki Takahashi, Department of Urology, Institute of Health Biosciences, The University of Tokushima Graduate School, 3-18-15 Kuramoto-cho, Tokushima 770-8503, Japan E-mail: takahashi.masayuki@tokushima-u.ac.jp

Key words: castration-resistant prostate cancer, tegafur-uracil, substitution of anti-androgen
( $p=0.019$ and $p=0.041$, respectively). In addition, in the patients with a lower TS expression, group B demonstrated a significantly longer TTP compared to group A $(p=0.018)$. There were no severe adverse events in either treatment group. After relapse from initial hormonal therapy, SOA combined with tegafururacil is effective and well tolerated. The TS mRNA expression level may be a predictive factor for this combination therapy.

\section{Introduction}

The management of patients with advanced prostate cancer who have relapsed after initial hormonal therapy is challenging. Some reports have demonstrated that substitution of anti-androgen (SOA) remains effective in this setting prior to introducing chemotherapies such as docetaxel, and it is mentioned as a treatment option in the European Association of Urology (EAU) 2012 guidelines. However, SOA has a relatively low PSA response rate (25-40\%) and a short duration of efficacy (4-6 months) (1-3). Therefore, we conducted this study to determine whether it would be more effective to add a mild oral chemotherapeutic agent to SOA.

Tegafur-uracil is an orally administered pro-drug of 5-fluorouracil (5-FU) with a cytostatic effect. Tegafur is metabolized into 5-FU in the liver and uracil is added to prevent 5-FU degradation. This drug has been approved in over 30 countries, including Japan. Adjuvant chemotherapy with tegafur-uracil has been demonstrated to be effective for several types of cancers. Patient survival after the complete resection of stage I lung adenocarcinoma is improved with tegafur-uracil administration (4). A significant survival benefit after surgery is also observed in patients with locally advanced gastric cancer when tegafur-uracil is used as an adjuvant therapy (5). Furthermore, 
based on the recent idea of 'tumor dormancy', tegafur-uracil has been given with hormonal therapy as an initial treatment for advanced prostate cancers with the expectation of cytostatic effect that results in a longer progression-free survival (6).

It has been reported that the expression level of enzymes involved in 5-FU metabolism may be correlated with 5-FU efficacy and clinical outcome. If the expression level of thymidylate synthase (TS), as a target enzyme of 5-FU, and dihydropyrimidine dehydrogenase (DPD), as a degrading enzyme, was low in gastrointestinal cancer tissues, there was a favorable response to 5-FU, leading to better prognosis (7-9). As 5-FU metabolic pathways were further investigated, it has also been indicated that thymidine phosphorylase (TP) (10) and orotate phosphoribosyltransferase (OPRT) (11) may be related to the 5-FU sensitivity.

It is known that the expression level of these 5-FU-related enzymes are quite different among various types of cancers (12). Invasive bladder cancer has higher TS expression, and it has a positive correlation with that of proliferation markers. Furthermore, patients with a high level of TS immunostaining had a significantly poorer prognosis in bladder cancer $(13,14)$. However, in prostate cancer, the expression level of these enzymes has not fully been examined, and the correlation of their expression level with stage, Gleason score or prognosis has not been investigated.

Therefore, we have conducted a multi-center prospective randomized phase II study to examine the effectiveness and safety of the combination therapy by comparing the combination therapy of SOA and tegafur-uracil with SOA alone for castration-resistant prostate cancer. In addition, we sought to examine whether the expression level of enzymes involved with 5-FU metabolism were correlated with the efficacy of the combination therapy and determine whether they would serve as predictive factors for the combination therapy.

\section{Materials and methods}

Patients. This study enrolled patients who were histologically confirmed to have prostate cancer and relapsed after initial hormonal therapy. Relapse was defined as at least three consecutive increases in PSA, the progression of measurable nodal or visceral lesions, or new metastatic lesions. Additional eligibility criteria included an Eastern Cooperative Oncology Group (ECOG) performance status (PS) $\leq 2$, a life expectancy of at least 3 months, adequate bone marrow, liver and renal function, and a normal electrocardiogram. Patients were ineligible if they had undergone a radical prostatectomy or radiation therapy for primary prostate cancer with curative intent, had an active infection, used phenytoin, had serious co-morbidity such as ileus, interstitial pneumonia and uncontrollable diabetes mellitus or had active concomitant cancer. All patients provided written informed consent to participate in this study. This study was approved by the institutional review board at each hospital and conducted in accordance with Good Clinical Practice guidelines and the Declaration of Helsinki.

Study design. This study was a randomized multicenter phase II study with 16 centers. Randomization was stratified based on prior hormonal therapy [LH-RH analogue/castration or maximum androgen blockage (MAB)], stage (non-metastatic or metastatic), and Gleason score $(\leq 6$ or $\geq 7$ ). If patients had disease progression after initial hormonal therapy, the anti-androgen was discontinued, and the patients were assessed for anti-androgen withdrawal syndrome (AWS). Thereafter, the patients were centrally randomly assigned in a 1:1 ratio to receive another anti-androgen alone (SOA, group A) or tegafur-uracil together with SOA (group B). The administered anti-androgens included bicalutamide, flutamide and chlormadinone acetate, which are available in Japan. Tegafur-uracil was orally administered at $200 \mathrm{mg}$ twice a day for patients with a body surface area (BSA) $\geq 1.35$ and $100 \mathrm{mg}$ three times a day for those with a BSA $<1.35$. Patients received continuous treatment until disease progression, death, unacceptable toxicity or withdrawal of consent for any reason. Subsequent anticancer therapy for patients with progressive disease was at the discretion of the patients and their physicians.

\section{Measurement of gene expression levels of the enzymes that are involved with 5-FU metabolism}

Laser capture microdissection (LCM). To exclusively obtain prostate cancer cells, a laser capture microdissection system (Carl Zeiss Microscopy GmbH, Jena, Germany) was utilized. Formalin-fixed paraffin-embedded (FFPE) blocks from prostatic needle biopsy specimens at diagnosis were serially sectioned and mounted onto non-coating slides and stained with hematoxylin and eosin $(\mathrm{H} \& \mathrm{E})$. Prostate cancer cells were precisely discriminated from benign and stromal cells by a pathologist. Tumor cell dissection was continued until at least a $1 \mathrm{~mm}^{2}$ area was obtained, and the dissected tumor cells were collected in sterile $0.5 \mathrm{ml}$ tube.

RNA extraction, complementary DNA (cDNA) preparation and amplification. Total RNA extraction and reverse transcription were performed using the Paradise Plus Whole Transcript Reverse Transcription (WT-RT) Reagent System (MDS Analytical Technologies, Sunnyvale, CA) according to the manufacturer's instructions. The amount and quality (i.e., RNA integrity number, RIN) of the extracted RNA were assessed with an Agilent 2100 Bioanalyzer using the Agilent RNA6000 Pico kit (Agilent Technologies, Santa Clara, CA). If the RIN was between 0 and 2, the RNA was considered to be degraded and not used for cDNA amplification. cDNA amplification was performed using the TaqMan PreAmp Master Mix kit (Life Technologies Inc., Carlsbad, CA) according to the manufacturer's instructions.

Quantitative reverse-transcription polymerase chain reaction ( $q$ PCR). A two-step TaqMan real-time qRT-PCR was performed with an ABI Prism 7900 machine (Life Technologies). Reactions were performed at $50^{\circ} \mathrm{C}$ for $2 \mathrm{~min}, 95^{\circ} \mathrm{C}$ for $10 \mathrm{~min}$ followed by 40 cycles of $95^{\circ} \mathrm{C}$ for $15 \mathrm{sec}$ and $60^{\circ} \mathrm{C}$ for $1 \mathrm{~min}$. Relative quantification was performed using the $\Delta \Delta \mathrm{C}_{\mathrm{t}}$ method in which the gene expression levels were normalized by using that of the $\beta$-actin gene as an endogenous control. Primers and TaqMan MGB probes for TS, DPD, OPRT, TP and $\beta$-actin were designed using the Primer Express ${ }^{\circledR}$ Software Version 3.0 (Life Technologies) with $71 \mathrm{bp}$ amplicons as follows: TS forward, 5'-GAATCACATCGAGCC ACTGAAA-3'; reverse, 5'-GAAGAATCCTGAGCTTTGGG AAA-3'; probe, 
Table I. Patient characteristics.

\begin{tabular}{|c|c|c|c|}
\hline Treatment arms & Group $A^{a}(n=26)$ & Group $B^{b}(n=26)$ & P-value \\
\hline Median age at study entry, years & $77(59-89)$ & $76.5(47-92)$ & 0.993 \\
\hline Median initial PSA, ng/ml & $98.45(5.8-15,168)$ & $311(4.7-3,454)$ & 0.462 \\
\hline Median PSA at study entry, ng/ml & $5.1(0.22-42.3)$ & $1.78(0.17-111.0)$ & 0.151 \\
\hline \multicolumn{4}{|l|}{ Stage at diagnosis } \\
\hline $\mathrm{B}$ & 5 & 4 & \multirow[t]{4}{*}{0.738} \\
\hline $\mathrm{C}$ & 6 & 4 & \\
\hline D1 & 1 & 3 & \\
\hline D2 & 14 & 15 & \\
\hline \multicolumn{4}{|l|}{ Gleason score } \\
\hline$\leq 6$ & 4 & 3 & \multirow[t]{3}{*}{$>0.999$} \\
\hline$=7$ & 3 & 4 & \\
\hline$\geq 8$ & 19 & 19 & \\
\hline \multicolumn{4}{|l|}{ Prior therapy } \\
\hline LH-RH analogue alone & 3 & 2 & \multirow[t]{2}{*}{$>0.999$} \\
\hline MAB & 23 & 24 & \\
\hline \multicolumn{4}{|l|}{ No. of prior anti-androgen } \\
\hline 1 & 20 & 22 & \multirow[t]{2}{*}{$>0.999$} \\
\hline 2 & 3 & 2 & \\
\hline \multicolumn{4}{|l|}{ Sequence of anti-androgens } \\
\hline BCL/FLT/CMA & 3 & 1 & \multirow[t]{8}{*}{0.449} \\
\hline CMA/BCL/FLT & 0 & 1 & \\
\hline BCL/FLT & 17 & 20 & \\
\hline BCL/CMA & 2 & 0 & \\
\hline FLT/BCL & 0 & 1 & \\
\hline CMA/BCL & 1 & 1 & \\
\hline Add BCL & 3 & 1 & \\
\hline Add CMA & 0 & 1 & \\
\hline
\end{tabular}

${ }^{\mathrm{a} G r o u p ~ g i v e n ~ s u b s t i t u t i o n ~ o f ~ a n t i-a n d r o g e n ~ a l o n e ; ~}{ }^{\mathrm{b}}$ group given tegafur-uracil and substitution of anti-androgen; MAB, maximum androgen blockade; BCL, bicalutamide; FLT, flutamide; CMA, chlormadinone acetate. The numbers in parentheses indicate range.

5'-CAGCT TCAGCGAGA AC-3'; DPD forward, 5'-TCTGGCTACCAGGCTATACAGTTT-3'; reverse, 5'-CAGC CTGTACAAGTGTCGGTTAT-3'; probe, 5'-AAACCCACCTG CCCAC-3'; OPRT forward, 5'-GGGACTACACTAGAGCAG CGGTTA-3'; reverse, 5'-GCCAGAAATAAAACCAACAACA AATT-3'; probe, 5'-AGTGCTCCTC AGCCAT-3'; TP forward, 5'-TCCTATATGCAGCCAGAGATGTG-3'; reverse, 5'-ACTG AGAATGGAGGCTGTGATG-3'; probe, 5'-TGGACAGCCT GCCAC-3'; $\beta$-actin forward, 5'-AAGGCCAACCGCGAG AAG-3'; reverse, 5'-ATAGCAACGTACATGGCTGGG-3'; probe, 5'-ACCCAGATCATGTTT-3'.

End-points and assessments. The primary end-point was the PSA response rate, which is defined as the percentage of patients with a $\geq 50 \%$ PSA decrease from baseline. The secondary end-points included time to PSA progression (TTP), adverse events (AEs), and the correlation of treatment efficacy with the mRNA expression of key enzymes involved in 5-FU metabolism. TTP was defined as the time interval between the start of therapy and the date of progression, which is defined as a rise in the PSA level three consecutive times and $\mathrm{a} \geq 50 \%$ increase from the nadir for PSA responders or three consecu- tive rising PSA levels and $\mathrm{a} \geq 25 \%$ increase from the baseline for PSA non-responders.

Patients were divided into two groups (low/high) according to the median expression level of each gene and PSA response rate and TTP were compared between two treatment groups.

Clinical assessments for safety, including clinical laboratory evaluations and any adverse events, were principally evaluated at baseline and every 4 weeks during the study and graded using the Common Terminology Criteria for Adverse Events of the National Cancer Institute, version 3.0.

Statistical methods. Categorical data were compared using the Wilcoxon test or the Fisher's exact test for proportion. The PSA response rate as a primary end-point was compared between two treatment arms with the Fisher's exact test. The PSA percent change from baseline to 12 weeks and the maximum PSA decline that occurred at any point after each treatment was compared using the Wilcoxon test. TTP analysis was performed using the Kaplan-Meier method and compared between two treatment arms using the log-rank test. Hazard ratios (HRs) with $95 \%$ confidence interval (CI) were calculated using the Cox proportional hazard model. The distribution of the mRNA 

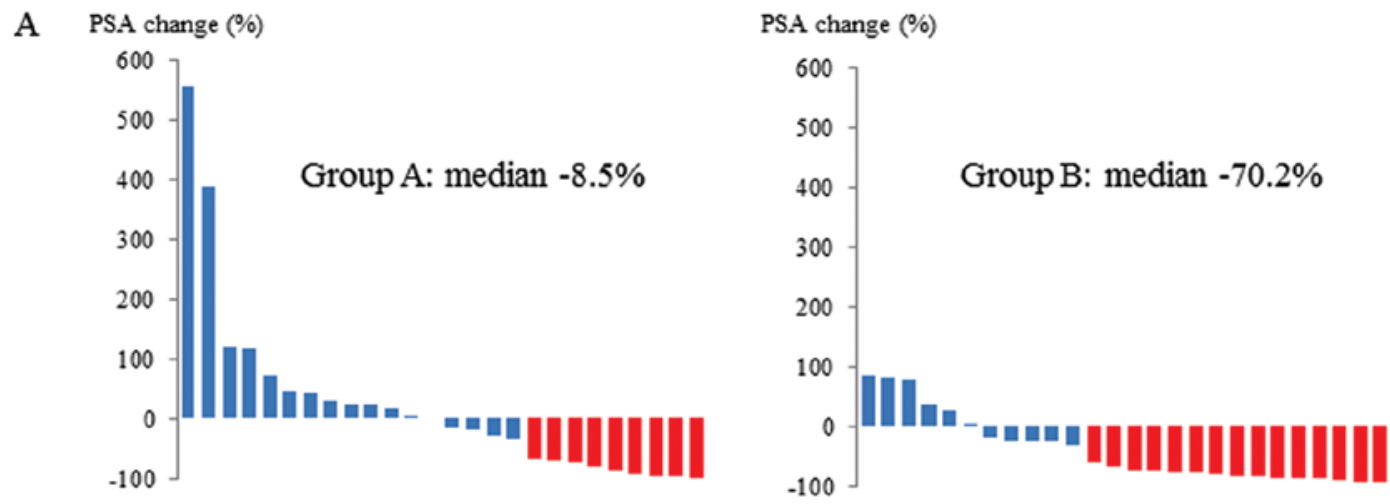

$<$ PSA change at 12 wks; $\mathrm{p}=0.116>$
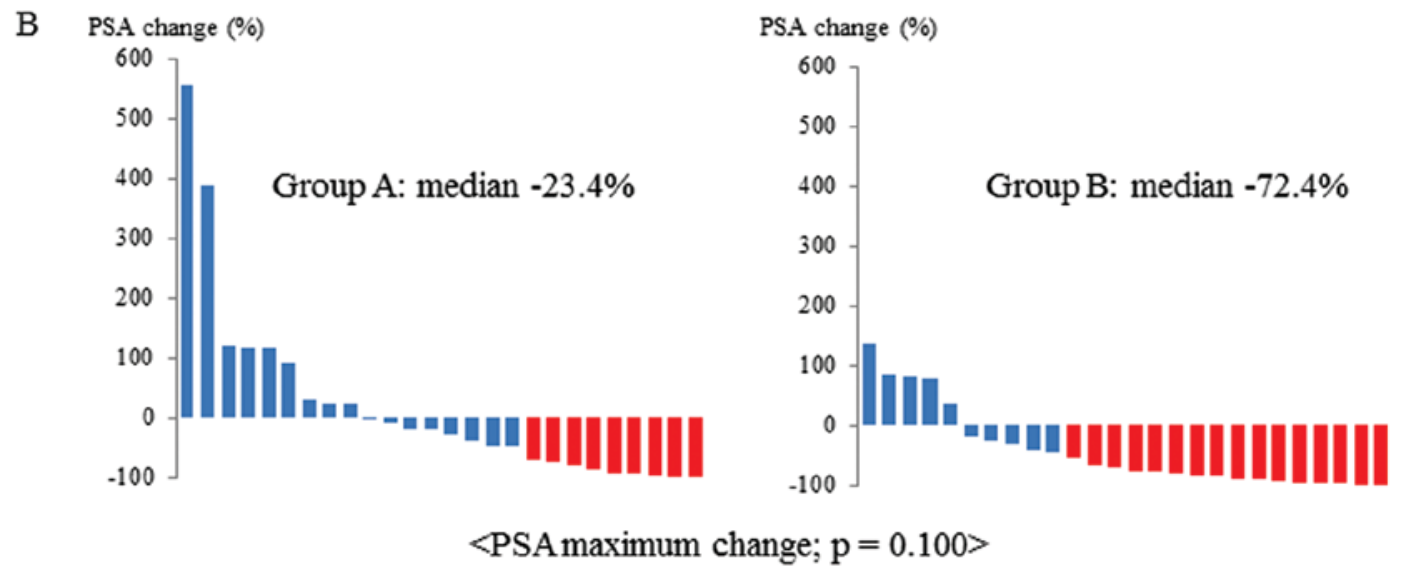

Figure 1. (A) Waterfall plots of a percent change in the PSA level from baseline to 12 weeks in group A (left) and B (right) are shown. Red bars show patients with $\geq 50 \%$ PSA decline. Patients with tegafur-uracil together with substitution of anti-androgen tended to have a greater PSA decrease (-8.5 vs. $-70.2 \%, p=0.116)$. (B) Waterfall plots of maximum decline in the PSA level during treatment in group A (left) and B (right) are shown. Red bars show patients with $\geq 50 \%$ PSA decline. Patients treated with tegafur-uracil and substitution of anti-androgen tended to have a greater PSA decrease during the entire treatment period (-23.4 vs. $-72.4 \%, \mathrm{p}=0.100)$.

expression levels of the enzymes in the two treatment arms was compared using a t-test. P-values were two-sided, and p-values $<0.05$ were considered statistically significant. The statistical analyses were performed with JMP $9^{\circledR}$ or SAS $9.1 .3^{\circledR}$ (SAS Institute Inc., Cary, NC, USA).

\section{Results}

Patients. Fifty-two patients were enrolled between February 2006 and November 2009 from 16 centers; 26 patients were randomly assigned to receive SOA alone (group A), and 26 patients were assigned to receive the combination therapy of SOA and tegafur-uracil (group B).

The majority of patients in each group (group A, 76.9\%; group B, 84.6\%) had received a maximum androgen blockade (MAB) with one prior anti-androgen at study enrollment. Only three patients (11.5\%) in group A and two patients $(7.7 \%)$ in group B had received two anti-androgens. There were no significant differences between the two treatment groups with regards to the patient characteristics including age at study entry, initial PSA level at diagnosis, PSA level at study entry, stage at diagnosis, Gleason score, prior therapy and number of anti-androgens (Table I). Bicalutamide was most frequently used as a prior treatment, which was often switched to flutamide after entry into the study (group A, 65.4\%; group B, 80.8\%).

PSA response rate. Nine of the 26 patients (34.6\%) in group A and 16 of the 26 patients $(61.5 \%)$ in group B had a PSA response. Although this difference was not statistically significant $(\mathrm{p}=0.095)$, patients who received tegafur-uracil together with SOA tended to have a higher PSA response rate (Table II).

The percent change in the PSA level from baseline. Half of the patients in group A had some PSA decline, and the median percent change in the PSA level from baseline to 12 weeks in group A was $-8.5 \%$. Seventy-seven percent of the patients in group B had a PSA decline, and the median percent change in the PSA level from baseline to 12 weeks in group B was $-70.2 \%$. Although there was no significant difference between the two treatment groups $(p=0.116)$, patients treated with tegafur-uracil together with SOA tended to have a greater PSA decline (Fig. 1A). 
Table II. PSA response and clinicopathological parameters.

\begin{tabular}{|c|c|c|c|c|c|}
\hline \multirow{2}{*}{$\begin{array}{l}\text { Clinical } \\
\text { parameters }\end{array}$} & \multicolumn{2}{|c|}{ Group $\mathrm{A}^{\mathrm{a}}$} & \multicolumn{2}{|c|}{ Group $\mathrm{B}^{\mathrm{b}}$} & \multirow[b]{2}{*}{ P-value } \\
\hline & No. & RR (\%) & No. & RR (\%) & \\
\hline All patients & 26 & 34.6 & 26 & 61.5 & 0.095 \\
\hline \multicolumn{6}{|l|}{ Age, years } \\
\hline$<77$ & 12 & 33.3 & 13 & 53.8 & 0.428 \\
\hline$\geq 77$ & 14 & 35.7 & 13 & 69.2 & 0.128 \\
\hline \multicolumn{6}{|l|}{ Stage } \\
\hline $\mathrm{B}, \mathrm{C}$ & 11 & 45.5 & 8 & 75.0 & 0.352 \\
\hline $\mathrm{D} 1, \mathrm{D} 2$ & 15 & 26.7 & 18 & 55.6 & 0.158 \\
\hline \multicolumn{6}{|l|}{ Gleason } \\
\hline$\leq 6$ & 4 & 75.0 & 3 & 66.7 & $>0.999$ \\
\hline$\geq 7$ & 22 & 27.3 & 23 & 60.9 & 0.036 \\
\hline
\end{tabular}

${ }^{a}$ Group given substitution of anti-androgen alone; 'broup given tegafur-uracil and substitution of anti-androgen; RR, response rate.

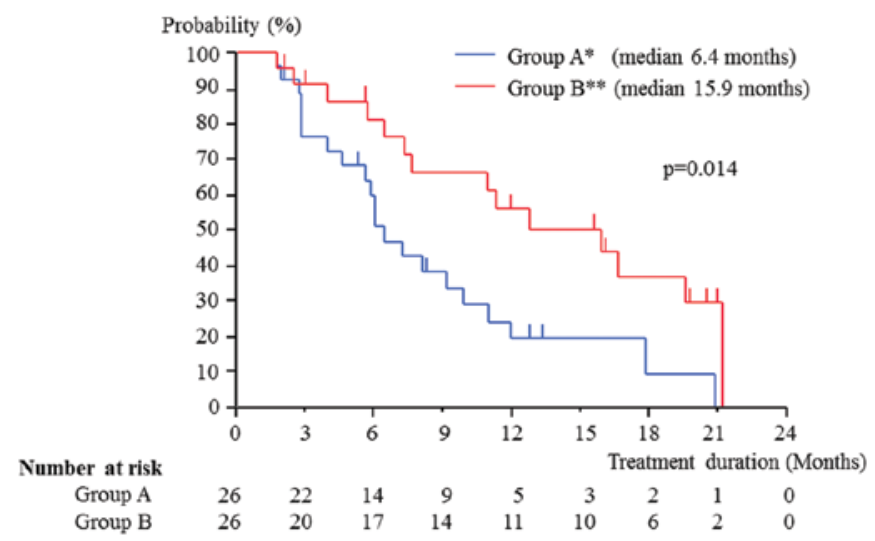

Figure 2. The time to PSA progression (TTP) for each treatment group is shown. The TTP in group B was significantly longer than that in group A (15.9 vs. 6.4 months, HR, $0.42 ; 95 \%$ CI: $0.20-0.85 ; \mathrm{p}=0.014$ ). Patients treated with tegafur-uracil and substitution of anti-androgen had a longer duration of PSA decline than patients treated with substitution of anti-androgen alone.

Table III. 5-FU metabolism-related mRNA expression level and clinical response.

\begin{tabular}{|c|c|c|c|c|c|c|c|c|}
\hline \multirow[b]{2}{*}{ Gene } & \multirow[b]{2}{*}{ Expression $\left(\times 10^{-2}\right)$} & \multicolumn{2}{|c|}{ Group $A^{a}$} & \multicolumn{2}{|c|}{ Group $\mathrm{B}^{\mathrm{b}}$} & \multirow[b]{2}{*}{ P-value } & \multicolumn{2}{|c|}{ TTP: Group $\mathrm{B}^{\mathrm{b}}$ vs. Group $\mathrm{A}^{\mathrm{a}}$} \\
\hline & & No. & $\mathrm{RR}(\%)$ & No. & $\mathrm{RR}(\%)$ & & $\mathrm{HR} \quad(95 \% \mathrm{CI})$ & P-value \\
\hline \multirow[t]{2}{*}{ TS } & $<0.95$ & 13 & 30.8 & 11 & 81.8 & 0.019 & $0.30(0.10-0.83)$ & 0.018 \\
\hline & $\geq 0.95$ & 12 & 33.3 & 13 & 46.2 & 0.688 & $0.48(0.13-1.49)$ & 0.213 \\
\hline \multirow[t]{2}{*}{ DPD } & $<1.45$ & 11 & 36.4 & 14 & 71.4 & 0.116 & $0.51(0.18-1.40)$ & 0.172 \\
\hline & $\geq 1.45$ & 14 & 28.6 & 10 & 50.0 & 0.403 & $0.35(0.08-1.16)$ & 0.103 \\
\hline \multirow[t]{2}{*}{ TP } & $<4.83$ & 14 & 42.9 & 10 & 80.0 & 0.104 & $0.38(0.13-1.04)$ & 0.057 \\
\hline & $\geq 4.83$ & 11 & 18.2 & 14 & 50.0 & 0.208 & $0.47(0.14-1.37)$ & 0.166 \\
\hline \multirow[t]{2}{*}{ OPRT } & $<4.89$ & 12 & 50.0 & 12 & 66.7 & 0.680 & $0.43(0.14-1.23)$ & 0.113 \\
\hline & $\geq 4.89$ & 13 & 15.4 & 12 & 58.3 & 0.041 & $0.36(0.10-1.08)$ & 0.072 \\
\hline
\end{tabular}

${ }^{a}$ Group given substitution of anti-androgen alone; broup given tegafur-uracil and substitution of anti-androgen; TTP, time to PSA progression; RR, response rate; HR, hazard ratio; TS, thymidylate synthase; DPD, dihydropyrimidine dehydrogenase; TP, thymidine phosphorylase; OPRT, orotate phosphoribosyltransferase.

The median maximum decline in PSA during treatment was $-23.4 \%$ in group A and $-72.4 \%$ in group B. Though this difference was also statistically non-significant $(\mathrm{p}=0.100)$, patients treated with tegafur-uracil together with SOA tended to have a greater PSA decline during the entire treatment period (Fig. 1B).

PSA response and clinicopathological parameters. The PSA response rate was compared between the two treatment groups in a subgroup divided according to each clinicopathological parameter, such as age ( $<77$ or $\geq 77$ ), stage (non-metastatic or metastatic), or Gleason score ( $\leq 6$ or $\geq 7$ ). The combination therapy of tegafur-uracil and SOA demonstrated a better PSA response rate than SOA alone in patients with higher Gleason score $(\mathrm{p}=0.036)$ (Table II).
Time to PSA progression (TTP). The TTP in group B was 15.9 months, which was significantly longer than that (6.4 months) in group A (HR, 0.42; 95\% CI: 0.20-0.85; p=0.014) (Fig. 2). Patients who received tegafur-uracil together with SOA had a longer duration of PSA decline than patients treated with SOA alone.

mRNA expression level of enzymes involved in 5-FU metabolism and PSA response rate. $\mathrm{mRNA}$ expression level of enzymes involved in 5-FU metabolism was measured in 49 patients because RNA of prostate cancer cells in the remaining three patients was degraded. The expression level distribution of genes encoding TS, DPD, OPRT and TP was not different between the two treatment groups at the diagnosis of prostate cancer ( $\mathrm{p}=0.866,0.776,0.694$ and 0.128 , respectively). The PSA 


\section{[Lower TS group $(<0.95)]$}

- Group A: median TTP 6.0 months

- Group B: median TTP 16.6 months

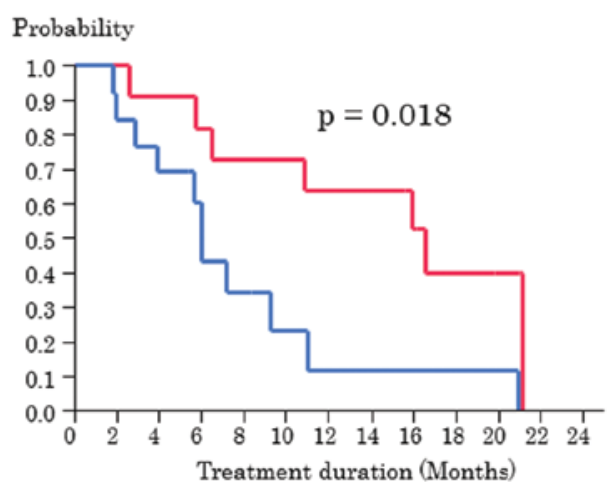

[Higher TS group $(\geq 0.95)]$

\section{- Group A: median TTP 6.4 months \\ - Group B: median TTP 11.3 months}

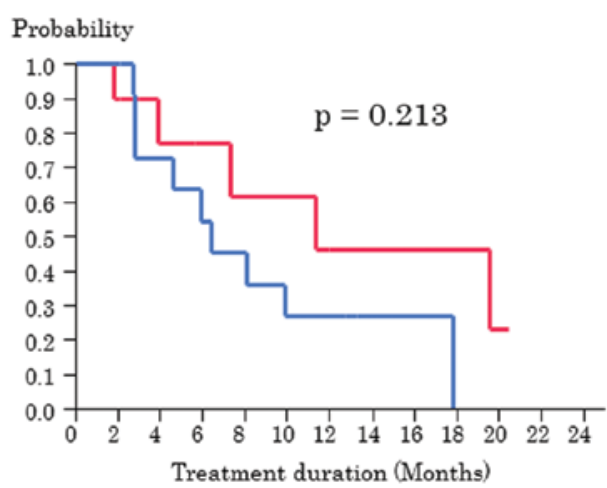

Figure 3. Left: Patients with a lower TS level. The patients in group B had a significantly longer time to PSA progression (TTP) than those in group A (16.6 vs. 6.0 months, HR, 0.30; 95\% CI: 0.10-0.83; p=0.018). Right: Patients with a higher TS level. The patients in group B had longer TTP than those in group A, but the difference was not statistically significant (11.3 vs. 6.4 months, HR, 0.48; $95 \%$ CI: 0.13-1.49; $\mathrm{p}=0.213$ ). Tegafur-uracil combined with substitution of anti-androgen may be more efficacious when prostate cancer cells express a low TS level.

response rate was compared between the two treatment groups in a subgroup divided by the median expression level of each gene. In patients with a lower TS expression and those with a higher OPRT expression, the combination therapy demonstrated significantly higher PSA response rate than the SOA alone ( $\mathrm{p}=0.019$ and 0.041 , respectively) (Table III).

TS expression level and time to PSA progression (TTP). When we examined the correlation of TTP and gene expression levels, patients with a lower TS level in group B had a significantly longer TTP than those in group A (16.6 vs. 6.0 months, HR, 0.30 ; 95\% CI: 0.10-0.83; $\mathrm{p}=0.018)$. However, the TTP was not significantly different in patients with a higher TS level (11.3 vs. 6.4 months; HR, 0.48; 95\% CI: 0.13-1.49; p=0.213) (Table III, Fig. 3). These data suggest that tegafur-uracil combined with SOA may be more effective when prostate cancer cells express a low TS level.

Safety. Most adverse events (AEs) were grade 1/2. Diarrhea (grade 1, 7.7\%) and ALT/AST elevation (grade 1/2, 7.7\%) were observed in group A. ALT/AST elevation (grade 1/2, 7.7\%), fatigue (grade $1 / 2,11.5 \%$ ), appetite loss (grade 2,3.8\%), nausea (grade 1,3.8\%) and elevation of total bilirubin (grade 3, 3.8\%) were reported in group B. Severe adverse events (AEs) were not observed in either treatment group. Although one patient in group B had a grade 3 elevation of total bilirubin, it spontaneously decreased to normal range after the discontinuation of tegafur-uracil. One patient in group A and seven patients in group B discontinued treatment. However, the treatment was discontinued due to patients request regardless of the mild AEs in all but one patient who had a grade 3 elevation in the total bilirubin.

\section{Discussion}

The majority of advanced prostate cancers relapse from initial hormonal therapy. Recently, docetaxel has been approved for castration-resistant prostate cancer as an effective chemotherapeutic agent compared with mitoxantrone in two pivotal studies $(15,16)$. In the group given docetaxel every three weeks with prednisone, the incidence of grades 3 and 4 neutropenia was relatively low (32\%), and febrile neutropenia was rare (3\%) (16). In the group given docetaxel and estramustine, the rate of grades 3-5 neutropenia was $16.1 \%$, and grades 3 and 4 neutropenic fevers were more common than those in the mitoxantrone group (15). In contrast, in a multicenter phase II trial of docetaxel and prednisone in Japan, the rate of grades 3 and 4 neutropenia was $93 \%$, and $16.3 \%$ of the patients had grades 3 or 4 febrile neutropenia, although a high response rate $(44.2 \%)$ was achieved (17).

Although docetaxel has also been the standard treatment for castration-resistant prostate cancer in Japan, the phenomenon of an aging population is remarkable compared with other countries, and there are quite a few older patients with prostate cancer, which may explain the higher rates of high grade neutropenia and febrile neutropenia. Therefore, more attention should be focused on Japanese patients with castration-resistant prostate cancer when docetaxel administration is considered.

SOA has often been attempted for patients who relapse from initial hormonal therapy in Japan. Although SOA is effective and could delay the introduction of docetaxel, its efficacy was relatively low with a PSA response rate of $25-40 \%$, and the efficacy duration was not long (4-6 months) (1-3).

Therefore, we conducted a multi-center randomized phase II study to examine the efficacy and safety of tegafururacil and SOA combination therapy compared with SOA alone. We have demonstrated a higher PSA response rate in the group given tegafur-uracil and SOA than the group given SOA alone. In addition, the PSA percent change from baseline to 12 weeks and the maximum PSA decline were greater for the combination therapy group. Although the data were not significantly different, they may be the result of a small sample size. Nevertheless, the TTP was significantly longer 
in the combination therapy group than in the SOA group, indicating a greater efficacy for the combination therapy.

When we examined the correlation of PSA response rate with clinicopathological parameters, the combination therapy demonstrated significantly higher PSA response rate than SOA alone in patients with higher Gleason score. This finding may suggest the combination therapy is more effective for more aggressive prostate cancer. However, as only a few patients had prostate cancer with Gleason score $\leq 6$ in this study, it should be confirmed by examining more patients.

We found that the combination therapy demonstrated a higher PSA response rate in patients with a lower TS expression or those with a higher OPRT expression among four 5-FU-related enzymes including TS, DPD, TP, and OPRT. In addition, in patients with a lower TS expression, the combination therapy demonstrated a significantly longer TTP than SOA alone. Inversely, Longley et al have demonstrated that TS overexpression confers increased resistance to TS inhibitors such as 5-FU by abrogating S-phase arrest in a breast cancer-derived cell line (18). In other words, DNA synthesis should be efficiently blocked by 5 -FU in the environments where the TS level is low. In addition, as a higher OPRT enzyme activity was observed in 5-FU-sensitive tissues than non-sensitive ones in in vitro chemosensitivity test in studies using human colorectal cancer tissues $(19,20)$, our results appear to be consistent with those of the aforementioned studies. Especially, the TS mRNA expression level in prostate cancer cells at diagnosis may predict the efficacy of the tegafur-uracil and SOA combination therapy.

The majority of patients were switched from bicalutamide to flutamide after they relapsed from the initial hormonal therapy in this study. We recently established a bicalutamide-resistant prostate cancer subline, CDX25R, and demonstrated that the combined treatment of 5-FU and hydroxyflutamide (OH-flutamide), the active metabolite of flutamide, had a synergistic effect on CDX25R cells (21). In addition, we found that $\mathrm{OH}$-flutamide decreased the expression of the E2F1 transcription factor followed by a decreased TS expression in CDX25R cells as the underlying mechanism. Because the TS expression in AR negative DU-145 cells was not affected by anti-androgens, it may be suggested that $\mathrm{OH}$-flutamide enhanced the growth-inhibitory activity of 5-FU in CDX25R cells by reducing the TS expression through the AR pathway.

There are several limitations to this study. Because this study began before the recommendations from the Prostate Cancer Clinical Trials Working Group (PCWG2) (22) were published, a measurable lesion by RECIST was not an eligibility criteria requirement, and the PSA change was particularly focused on as the treatment efficacy in this study. In addition, 23 of the 52 evaluable patients (44\%) had a PSA level lower than $2 \mathrm{ng} / \mathrm{ml}$ at the start of the study. Because an early PSA increase does not always indicate disease progression in castration-resistant prostate cancer, some patients in this study may not have reflected true disease progression and shown actual treatment efficacy. However, because tegafur-uracil is a mild chemotherapeutic agent with a cytostatic effect, it is unlikely to show a significant shrinkage in the target lesions. Some patients may request a change in treatment prior to their PSA levels reaching $2 \mathrm{ng} / \mathrm{ml}$ in a clinical setting. Nonetheless, the results in this study warrant further examination with more patients because this study was conducted in a prospective randomized fashion.

The tegafur-uracil and SOA combination therapy did not cause severe adverse events and proved to be safe although more patients discontinued the combination therapy due to mild adverse events.

We conclude that this combination therapy may be a treatment option before the introduction of chemotherapies such as docetaxel, particularly when more severe adverse events are expected in older patients. However, further investigation is required with more patients to elucidate whether the combination therapy could actually be an effective treatment option.

\section{Acknowledgements}

We thank the investigators at the following participating institutions: The University of Tokushima Graduate School, Sanuki Municipal Hospital, Tokushima Municipal Hospital, Tsurugi Municipal Hospital, Tokushima Prefectural Central Hospital, Tokushima Prefecture Naruto Hospital, Kamei Hospital, Oe Kyodou Hospital, Anan Central Hospital of the Medical Association, Tokushima Prefectural Miyoshi Hospital, Kaisei Hospital, National Hospital Organization Zentsuji National Hospital, Japanese Red Cross Takamatsu Hospital, Yashima General Hospital, Japanese Red Cross Kochi Hospital and Kochi Takasu Hospital. This study was conducted under financial support of TAIHO Pharmaceutical Co. Ltd. (trial registration ID: UMIN000009181).

\section{References}

1. Kojima S, Suzuki H, Akakura K, Shimbo M, Ichikawa T and Ito $\mathrm{H}$ : Alternative antiandrogens to treat prostate cancer relapse after initial hormone therapy. J Urol 171: 679-683, 2004.

2. Okihara K, Ukimura O, Kanemitsu N, Mizutani Y, Kawauchi A and Miki T: Clinical efficacy of alternative antiandrogen therapy in Japanese men with relapsed prostate cancer after first-line hormonal therapy. Int J Urol 14: 128-132, 2007.

3. Suzuki H, Okihara K, Miyake H, et al: Alternative nonsteroidal antiandrogen therapy for advanced prostate cancer that relapsed after initial maximum androgen blockade. J Urol 180: 921-927, 2008.

4. Kato $\mathrm{H}$, Ichinose $\mathrm{Y}$, Ohta $\mathrm{M}$, et al: A randomized trial of adjuvant chemotherapy with uracil-tegafur for adenocarcinoma of the lung. N Engl J Med 350: 1713-1721, 2004.

5. Nakajima T, Kinoshita T, Nashimoto A, et al: Randomized controlled trial of adjuvant uracil-tegafur versus surgery alone for serosa-negative, locally advanced gastric cancer. Br J Surg 94: 1468-1476, 2007.

6. Kuriyama M, Takahashi Y, Sahashi M, et al: Prospective and randomized comparison of combined androgen blockade versus combination with oral UFT as an initial treatment for prostate cancer. Jpn J Clin Oncol 31: 18-24, 2001.

7. Ichikawa W, Uetake H, Shirota Y, et al: Combination of dihydropyrimidine dehydrogenase and thymidylate synthase gene expressions in primary tumors as predictive parameters for the efficacy of fluoropyrimidine-based chemotherapy for metastatic colorectal cancer. Clin Cancer Res 9: 786-791, 2003.

8. Salonga D, Danenberg KD, Johnson M, et al: Colorectal tumors responding to 5-fluorouracil have low gene expression levels of dihydropyrimidine dehydrogenase, thymidylate synthase, and thymidine phosphorylase. Clin Cancer Res 6: 1322-1327, 2000.

9. Suda Y, Kuwashima Y, Tanaka Y, Uchida K and Akazawa S: Immunohistochemical detection of thymidylate synthase in advanced gastric cancer: a prognostic indicator in patients undergoing gastrectomy followed by adjuvant chemotherapy with 5-fluoropyrimidines. Anticancer Res 19: 805-810, 1999. 
10. Metzger R, Danenberg K, Leichman CG, et al: High basal level gene expression of thymidine phosphorylase (platelet-derived endothelial cell growth factor) in colorectal tumors is associated with nonresponse to 5-fluorouracil. Clin Cancer Res 4: 2371-2376, 1998.

11. Ichikawa W, Uetake H, Shirota $\mathrm{Y}$, et al: Both gene expression for orotate phosphoribosyltransferase and its ratio to dihydropyrimidine dehydrogenase influence outcome following fluoropyrimidine-based chemotherapy for metastatic colorectal cancer. Br J Cancer 89: 1486-1492, 2003.

12. Fukushima M, Morita M, Ikeda K and Nagayama S: Population study of expression of thymidylate synthase and dihydropyrimidine dehydrogenase in patients with solid tumors. Int J Mol Med 12: 839-844, 2003.

13. Hugosson J, Bergdahl S, Carlsson G, Frosing R, Norlen L and Gustavsson B: Effects of intravesical instillation of 5-fluorouracil and interferon in patients with recurrent superficial urinary bladder carcinoma. A clinical and pharmacodynamic study. Scand J Urol Nephrol 31: 343-347, 1997.

14. Nomura T, Nakagawa M, Fujita Y, Hanada T, Mimata $H$ and Nomura Y: Clinical significance of thymidylate synthase expression in bladder cancer. Int J Urol 9: 368-376, 2002.

15. Petrylak DP, Tangen CM, Hussain MH, et al: Docetaxel and estramustine compared with mitoxantrone and prednisone for advanced refractory prostate cancer. N Engl J Med 351: 1513-1520, 2004

16. Tannock IF, de Wit R, Berry WR, et al: Docetaxel plus prednisone or mitoxantrone plus prednisone for advanced prostate cancer. N Engl J Med 351: 1502-1512, 2004.
17. Naito S, Tsukamoto T, Koga H, et al: Docetaxel plus prednisolone for the treatment of metastatic hormone-refractory prostate cancer: a multicenter Phase II trial in Japan. Jpn J Clin Oncol 38: 365-372, 2008.

18. Longley DB, Ferguson PR, Boyer J, et al: Characterization of a thymidylate synthase (TS)-inducible cell line: a model system for studying sensitivity to TS- and non-TS-targeted chemotherapies. Clin Cancer Res 7: 3533-3539, 2001.

19. Isshi K, Sakuyama T, Gen T, et al: Predicting 5-FU sensitivity using human colorectal cancer specimens: comparison of tumor dihydropyrimidine dehydrogenase and orotate phosphoribosyl transferase activities with in vitro chemosensitivity to 5-FU. Int J Clin Oncol 7: 335-342, 2002.

20. Fujii R, Seshimo A and Kameoka S: Relationships between the expression of thymidylate synthase, dihydropyrimidine dehydrogenase, and orotate phosphoribosyltransferase and cell proliferative activity and 5-fluorouracil sensitivity in colorectal carcinoma. Int J Clin Oncol 8: 72-78, 2003.

21. Kawabata R, Oie S, Oka T, Takahashi M, Kanayama H and Itoh K: Hydroxyflutamide enhances cellular sensitivity to 5-fluorouracil by suppressing thymidylate synthase expression in bicalutamide-resistant human prostate cancer cells. Int $\mathbf{J}$ Oncol 38: 665-676, 2011.

22. Scher HI, Halabi S, Tannock I, et al: Design and end-points of clinical trials for patients with progressive prostate cancer and castrate levels of testosterone: recommendations of the Prostate Cancer Clinical Trials Working Group. J Clin Oncol 26: 1148-1159, 2008. 